\title{
THE MICROMECHANICAL RESONANT SWITCH ("RESOSWITCH")
}

\author{
Yang Lin, Wei-Chang Li, Zeying Ren and Clark T.-C. Nguyen \\ Department of Electrical Engineering and Computer Sciences \\ University of California at Berkeley, Berkeley, California 94720, USA
}

\begin{abstract}
A micromechanical switch, dubbed the "resoswitch", has been demonstrated that harnesses the resonance and nonlinear dynamical properties of its mechanical structure to greatly increase switching speed and cycle count (even under hot switching), and lower the needed actuation voltage, all by substantial factors over existing RF MEMS switches. The device comprises a wine-glass mode disk resonator driven hard via a $2.5 \mathrm{~V}$ amplitude ac voltage at its $61-\mathrm{MHz}$ resonance frequency so that it impacts electrodes along an orthogonal switch axis, thereby closing a switch connecting a $10 \mathrm{~V}$ source to the switch electrode. The $61-\mathrm{MHz}$ operating frequency corresponds to a switching period of $16 \mathrm{~ns}$ with an effective rise time of $<4 \mathrm{~ns}$, which is more than 200 times faster than the $\mu$ s-range switching speeds of the fastest RF MEMS switches. Furthermore, since the voltage source is on during switching, the switch essentially hot switches with a demonstrated lifetime exceeding 16.5 trillion cycles without failure, but with some observed degradation.
\end{abstract}

\section{INTRODUCTION}

RF MEMS switches operating at RF to millimeter-wave frequencies substantially outperform p-i-n diode and field-effect transistor (FET) counterparts in insertion loss, isolation, and switch figure of merit (FOM). Unfortunately, their much slower switching speeds (e.g., 1-15 $\mu$ s versus the $0.16-1 n s$ [1] of FET's) and cycle lifetimes on the order of 100 billion cycles (for the good ones) relegates them mainly to antenna switching, reconfigurable aperture, and instrumentation applications, and precludes them from much higher volume applications, such as switched-mode power amplifiers and power converters.

Indeed, the benefits afforded to switched-mode power applications that would ensue if the transistors they presently employ were replaced by switches with FOM's on the order of those exhibited by RF MEMS switches would be enormous. For example, switched-mode power amplifiers that ideally should be able to achieve $100 \%$ drain efficiency presently cannot attain such values in practical implementations, in part because the transistors they use for switching exhibit large input capacitors (for small "on" resistances) and are often limited in the voltages they can support. MEMS switches, being made of metal, have very small "on” resistances and would be able to support higher voltages. However, if they are to be used in switched-mode power applications, their actuation voltages would need to be lowered substantially (from $>50 \mathrm{~V}$ down to the single-digit volt range), their speeds would need to be much higher (e.g., ns switching times), and their reliability enhanced substantially, since typical power amplifier and converter applications would require cycle counts in the 100's of quadrillions.

Pursuant to achieving a switch suitable for power amplifier and converter applications, this work demonstrates a micromechanical switch, dubbed the "resoswitch", that harnesses the resonance and nonlinear dynamical properties of its mechanical structure to greatly increase switching speed and cycle count (even under hot switching), and lower the needed actuation voltage, all by substantial factors over existing RF MEMS switches. The device comprises a wine-glass mode disk resonator driven hard via a $2.5 \mathrm{~V}$ amplitude ac voltage at its 61-MHz resonance frequency so that it impacts electrodes along an orthogonal switch axis, thereby closing a

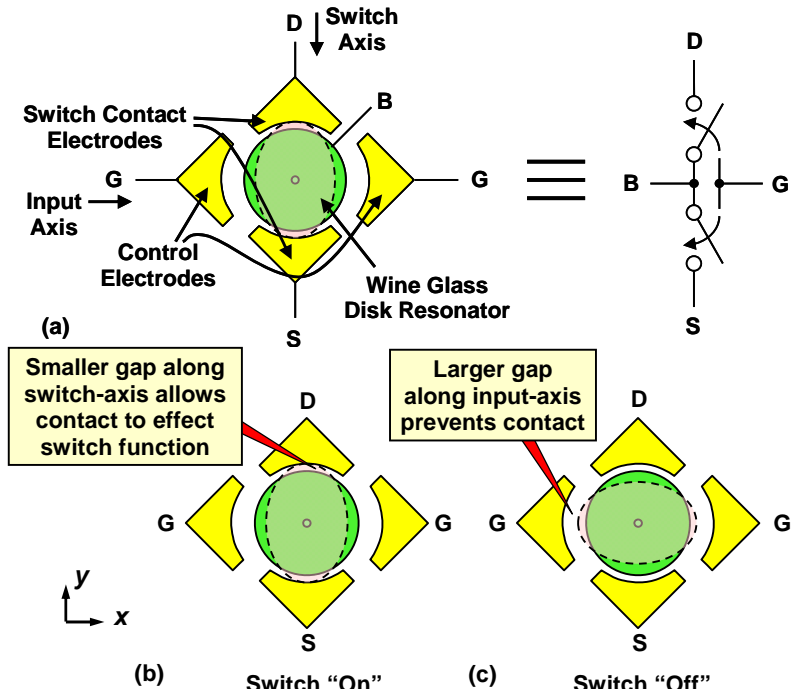

Fig. 1: Schematics showing (a) the physical structure of the micromechanical resoswitch, identifying its ports and equating it to a functional equivalent circuit; (b) its "on"; and (c) its "off" states.

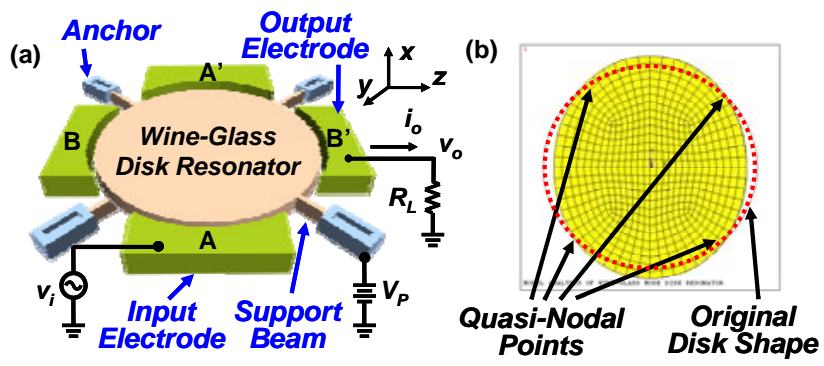

Fig. 2: (a) Perspective-view schematic of a micromechanical wine-glass-mode disk resonator in a typical two-port bias and excitation configuration (where A,A' are electrically connected, as are $B, B$ '); (b) ANSYS simulated wine-glass mode shape.

switch connecting a $10 \mathrm{~V}$ source to the switch electrode. The $61-\mathrm{MHz}$ operating frequency corresponds to a switching period of $16 \mathrm{~ns}$ with an effective rise time of $<4 \mathrm{~ns}$, which is more than 200 times faster than the $\mu$ s-range switching speeds of the fastest RF MEMS switches. Furthermore, since the voltage source is on during switching, the switch essentially hot switches with a demonstrated lifetime exceeding 16.5 trillion cycles without failure, but with some observable degradation.

\section{RESOSWITCH STRUCTURE AND OPERATION}

Fig. 1 presents schematics describing the structure and operation of one simple rendition of a resoswitch that comprises a capacitively-transduced wine-glass disk micromechanical resonator [2] (c.f., Fig. 2) made in a conductive material (preferably, a metal) and surrounded by four electrodes, two of which are situated along an indicated input axis having larger electrode-to-resonator gaps than their counterparts along an orthogonal switch axis. To operate the switch, an ac input voltage at the wine-glass mode disk resonance frequency is applied to the input electrodes (along the input 
(a) Time Domain Response

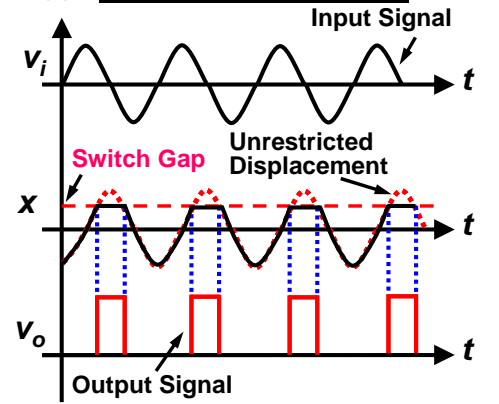

(b) Freq. Response

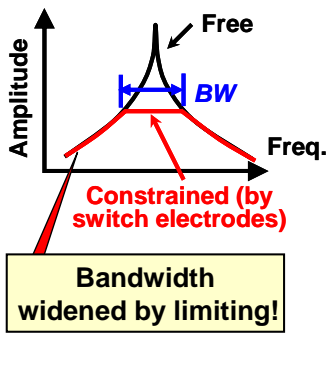

Fig. 3: (a) Time domain waveforms describing the relationships between the input signal, the ensuing displacement $\left(90^{\circ}\right.$ phase-shifted from the input), and the resulting output signal along the switch axis. In the displacement waveform, the red dotted curve indicates the displacement that would ensue if there were no limiting. (b) Expected frequency response illustrating bandwidth widening effect by impact limiting.

axis), electrically forcing the disk into the wine-glass mode shape delineated by the dotted curve in Fig. 1(a). As the resonance amplitude rises, the disk eventually impacts the (closer) electrodes along the switch axis, as shown in Fig. 1(b). This then ideally steals energy from the disk, effectively limiting its amplitude so that when the disk elongates along the input axis on the next cycle, it does not impact the (more distant) input electrodes, as shown in Fig. 1(c). Essentially, in this structure, control electrodes more distant from the disk are used to the drive the disk into its resonance mode shape (indicated by the dotted curve), where at sufficient amplitude it impacts closer electrodes along the orthogonal axis, closing the switch at a frequency equal to the resonance frequency of the disk. Because the input is applied at the resonance frequency of the disk, the required input voltage amplitude that effects switching along the switch axis is quite small, on the order of $1-3 \mathrm{~V}$, which is much smaller than the $>50 \mathrm{~V}$ required by most RF MEMS switches.

The utility of a switch operating at resonance is immediately apparent upon recognition that some of the highest volume applications of switches, such as switched-mode power converters [3] and amplifiers [4], operate in a mode where their switches switch continuously with essentially constant period within specified bandwidths. The use of the present micromechanical resoswitch in place of the switching transistor in these applications (c.f., Fig. 4) stands to greatly enhance performance by allowing the use of much higher voltages (e.g., $10 \mathrm{~V}$ vs. the $1 \mathrm{~V}$ limit of conventional CMOS) [5], which raises the efficiency of switched-mode power amplifiers and raises the voltage levels achievable by power converters.

Of course, the cycle count of the resoswitch would need to be much larger than so far achieved by RF MEMS switches in order to be useful for such power applications. In this regard, the reliability of the present resoswitch benefits from two major advantages: 1) the stiffness of its actuating disk resonator is $1.15 \times 10^{6} \mathrm{~N} / \mathrm{m}$, which is several orders larger than that of a conventional RF MEMS switch, so provides a substantially larger restoring force with which to overcome sticking forces; and 2) the energy stored via resonance vibration of the device provides a momentum that further increases the effective restoring force. The use of such a large spring restoring force is in turn made possible by resonance operation, under which the displacement of the actuator is $Q$ times larger than off-resonance, allowing a mere $1-3 \mathrm{~V}$ amplitude drive voltage to generate impacting switch axis amplitudes.

Fig. 3 presents the expected time domain waveforms generated by a direct contact version of the resoswitch under ideal operation. Here, the duty cycle of the output waveform is controlled
Table 1: On-Chip Switch Comparison

\begin{tabular}{|c|c|c|c|}
\hline Parameter & FET & RF MEMS & Resoswitch \\
\hline Actuation Voltage & $1-3 \mathrm{~V}$ & $20-80 \mathrm{~V}$ & $2.5 \mathrm{~V}$ \\
\hline Maximum Voltage & $1-3 \mathrm{~V}$ & $>100 \mathrm{~V}$ & $>100 \mathrm{~V}$ \\
\hline Switching Time & $0.16-1 \mathrm{~ns}[1]$ & $1-300 \mu \mathrm{s}$ & $\sim 4 \mathrm{~ns}$ \\
\hline Life Time & Very Long & 100 Billion [9] & $>16.5$ Trillion \\
\hline On Resistance & $0.5 \Omega[6]$ & $0.1-1 \Omega[9]$ & $0.1-1 \Omega *$ \\
\hline Input Capacitance & $20 \mathrm{pF}[6]$ & $1-10 \mathrm{fF}[10]$ & $20-30 \mathrm{fF}$ \\
\hline FOM $=1 /\left(2 \pi R_{o n} C_{\text {off }}\right)$ & $590 \mathrm{GHz}[7]$ & $63 \mathrm{THz}$ & $>30 \mathrm{THz}^{*}$ \\
\hline
\end{tabular}

* Potentially achievable by a metal version.

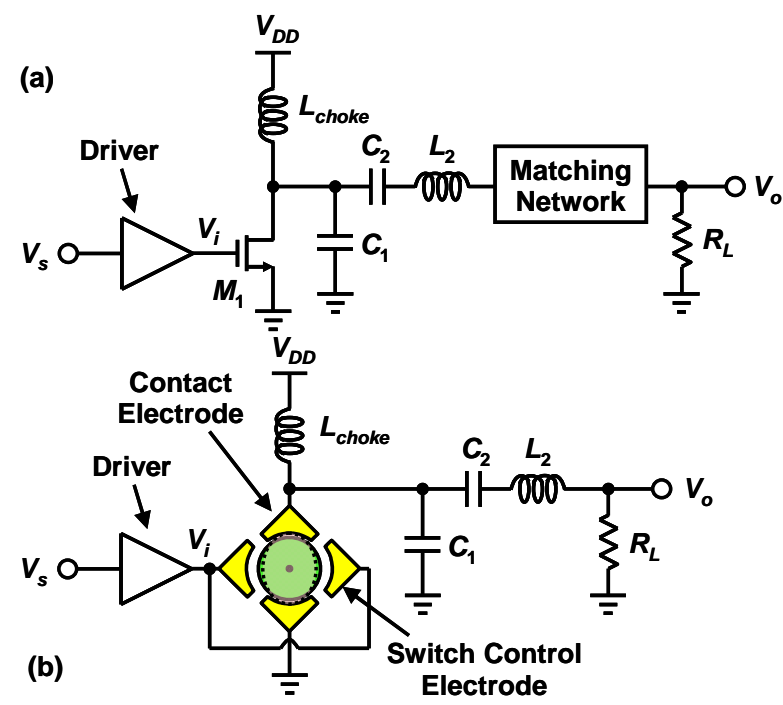

Fig. 4: Circuit topologies of (a) a conventional Class E amplifier using a transistor switch device; and (b) one simplified rendition of the proposed Class E amplifier utilizing the vibrating micromechanical resonator switch described herein. (The dashed line in (b) indicates how the resoswitch contorts to make contact.).

by how hard the resoswitch is driven. In particular, when the resoswitch is driven softly, so that it only barely touches the switch axis electrodes, the duty cycle is very small. When driven very hard, the duty cycle rises closer to its $50 \%$ maximum.

Not only is duty cycle adjustable, but so is the bandwidth of the resoswitch. In particular, as shown in Fig. 3(b), the bandwidth over which impacting occurs can also be controlled by the amplitude of the ac input voltage. In effect, the larger the input voltage amplitude, the lower is the frequency of first limiting on the frequency characteristic of the device, and the higher the frequency of last limiting. This bandwidth-widening is a nonlinear dynamical effect that simultaneously allows high $Q$ along the input axis, which lowers the required input ac voltage; while also allowing a wide effective bandwidth along the switch (or output) axis. The availability of simultaneous high- $Q$ and wide bandwidth obviously benefits transmit power amplifier applications in communications, since it permits wide frequency modulations on the transmitted signal while simultaneously lowering the input capacitance for a given input signal amplitude needed to operate the device.

\section{RESOSWITCH ADVANTAGES}

Table 1 compares the described micromechanical resoswitch with transistor FET and RF MEMS switch counterparts, showing clear advantages in maximum voltage and input capacitance over 
transistor FET's, and in speed over RF MEMS switches. Although the device-to-device comparisons are already favorable for the resoswitch, the advantages provided by the described resoswitch are perhaps best elucidated in the context of an application. To this end, Fig. 4 shows the circuit topologies of (a) a conventional Class E power amplifier (PA) using a transistor switch device; and (b) one simplified rendition of the same amplifier utilizing the vibrating micromechanical resonant switch demonstrated in this work. The use of the present micromechanical resoswitch in place of the switching transistor in this application stands to greatly enhance performance in the following ways:

1) It allows the use of much higher voltages (e.g., $10 \mathrm{~V}$ vs. the 1-3V limit of conventional CMOS) [5]. This then allows the resoswitch rendition to directly drive the $50 \Omega$ load, and thereby dispense with the lossy matching network needed by the transistor version. (Because of maximum voltage limitations, the voltage provided by the transistor FET is too small to deliver the required $1-2 \mathrm{~W}$ of power into $50 \Omega$. Thus, a matching network is required to lower the impedance driven by the transistor to $\sim 2 \Omega$.) Removal of the matching network and raising the driven impedance from $2 \Omega$ to $50 \Omega$ can raise the efficiency of a Class E power amplifier by as much as $23 \%$.

2) The use of the resoswitch further allows the same or smaller "on" resistance than its FET counterpart, but with substantially smaller input capacitance, e.g., only 20fF for the resoswitch versus $20 \mathrm{pF}$ for a CMOS PA switch-a $1000 \times$ difference that removes much of the input power that would otherwise be needed to drive the PA. Again, better efficiency ensues.

\section{EXPERIMENTAL RESULTS}

To demonstrate the resoswitch, doped polysilicon wine-glass mode disk resonators based on the design and fabrication process of [2] were employed. Fig. 5(a) presents the SEM of one of the $61-\mathrm{MHz}$ wineglass disk resonators used in this work, with a zoom-in shot in (b) showing the tiny gap between the disk and its switch electrode. For most power amplifier and converter applications, the resoswitch should be constructed of metal, not polysilicon, to reduce its contact and series resistance. The use of doped polysilicon in this work does compromise resoswitch performance, especially with regards to the switch "on" resistance, which is dominated by the $1 \mathrm{k} \Omega$ parasitic resistance $R_{p}$ of its polysilicon leads and interconnects. Nevertheless, it still allows demonstration of practically all other important resoswitch performance parameters. It should be noted that, despite its high series resistance, the polysilicon version of the resoswitch is actually still quite applicable for use in low current drain switch-mode on-chip dc-to-dc power converters (i.e., charge pumps), such as needed to supply the large dc-bias voltages often required by capacitively transduced vibrating RF MEMS devices [11].

For simplicity in this early demonstration, the strategy of using different electrode-to-disk spacings along the input and switch axes shown in Fig. 1 was not used in this implementation. Rather, the electrode-to-resonator gap spacings for both axes were $100 \mathrm{~nm}$ for direct contact switches, in which the conductive disk and electrode materials actually make electrical contact; and about $97 \mathrm{~nm}$ for capacitive switches, in which a thin layer of oxide exists over conductive surfaces that prevents electrical contact, but still allows switching through the large capacitance that results when the disk impacts its switch electrodes. For the direct contact version of the resoswitch, one obvious consequence of the use of identical input and switch axis electrode-to-resonator gaps is that the input gets shorted to the disk during operation, which then prevents deployment of the device in the fashion shown in Fig. 4, which would

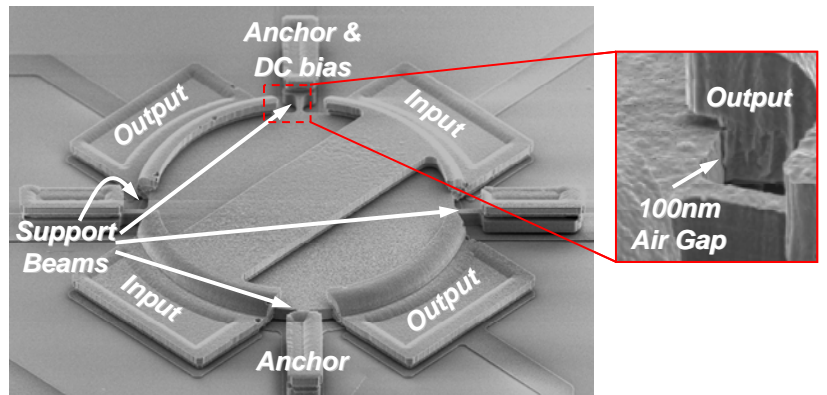

Fig. 5: (a) SEM of the polysilicon resoswitch demonstration vehicle used in this work, which essentially comprises a wine-glass disk resonator with properly spaced and positioned electrodes; and (b) zoom-in on the electrode-to-resonator gap of the device along the switch axis.

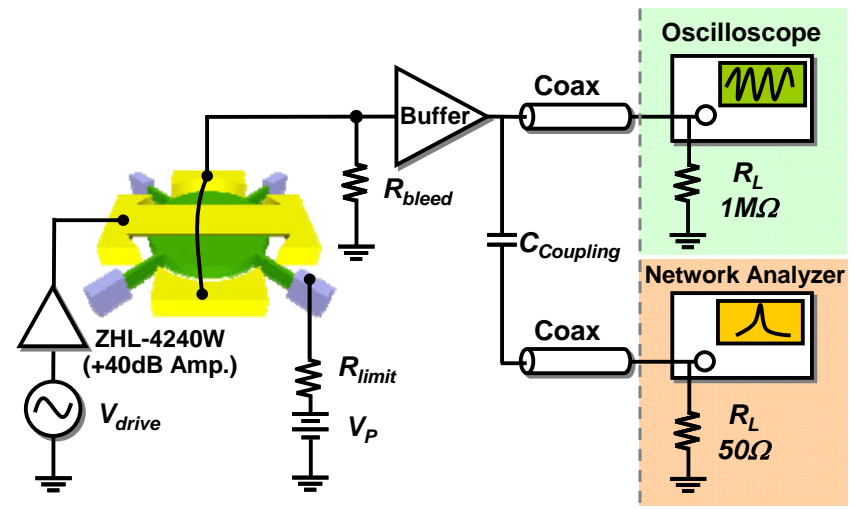

Fig. 6: Test set-up used to evaluate the micromechanical resoswitch.

otherwise be preferred.

To circumvent this problem, the test set-up used to measure resoswitch performance, depicted in Fig. 6, uses a less practical configuration, but one still very valid for evaluation of switch performance. Here, a dc-bias voltage $V_{P}$ is applied to the disk structure that is effectively applied to the output node when the switch closes (i.e., comes "on") in the fashion shown in Fig. 1. As shown, this circuit allows both time domain (i.e., oscilloscope) and frequency domain (i.e., network analyzer) observation of the resoswitch output. The output buffer used in this circuit effectively removes the $80 \mathrm{pF}$ of coaxial capacitance that would otherwise load the output node of the resoswitch and greatly reduce the signal level due to $3 \mathrm{~dB}$ bandwidth roll-off. The output buffer, however, is not perfect, as it still loads the output node of the resoswitch with about $4 \mathrm{pF}$. This is large enough to round out the corners of the expected output square wave (c.f., Fig. 3) so that it looks more sinusoidal.

Fig. 7 and Fig. 8 present the oscilloscope waveform and swept frequency response spectrum, respectively, of the direct contact resoswitch, verifying switching operation, impact limiting, and also a bandwidth-widening effect that could be harnessed for wider-band power amplifier applications that should be accessible to future metal renditions of the resoswitch. Switching clearly occurs when the frequency response grows suddenly and limits with a "flat top", as shown on Fig. 8. This occurs when the voltage amplitude reaches 2.5V. The measured output signal on Fig. 7 has a peak-to-peak amplitude of about $1 \mathrm{~V}$, which is the value expected when considering attenuation via the finite $3 \mathrm{~dB}$ bandwidth of the measurement circuit of Fig. 6, and when considering the voltage divider formed by the parasitic polysilicon interconnect resistance $R_{p}$ and the bleed resistor $R_{\text {bleed }}$. The signal is not quite a square wave due to bandwidth limitations of the measurement circuit, but the amplitude is correct. To emphasize this point, Fig. 7 also includes a SPICE simulated waveform that includes the effects of $1.1 \mathrm{k} \Omega$ of parasitic 


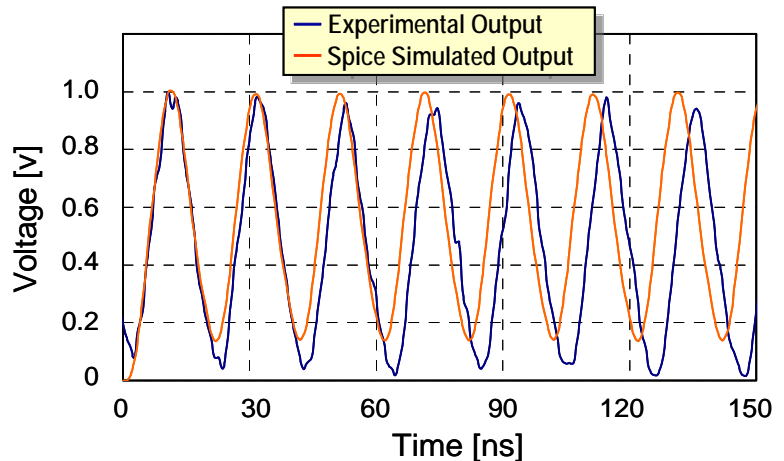

Fig. 7: Oscilloscope (i.e., time domain) waveform and SPICE simulated prediction seen at the resoswitch output node of Fig. 5 when driven by a resonance input signal with $2.5 \mathrm{~V}$ amplitude.

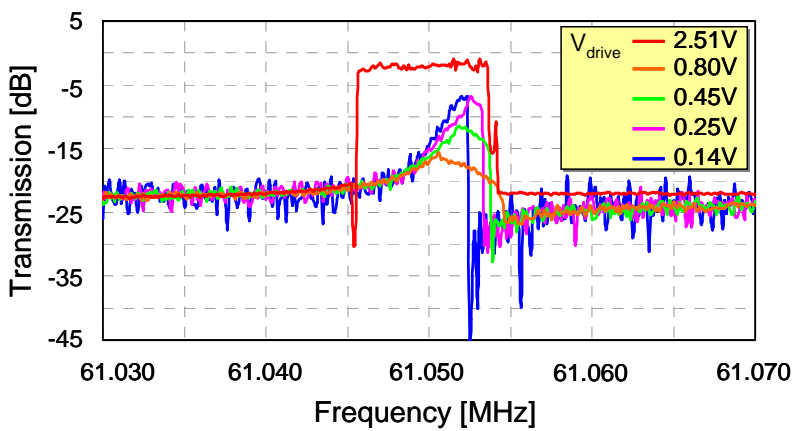

Fig. 8: Frequency response (in vacuum) as measured by a network analyzer of the direct contact version of the resoswitch for varying resonance input ac voltage amplitudes.

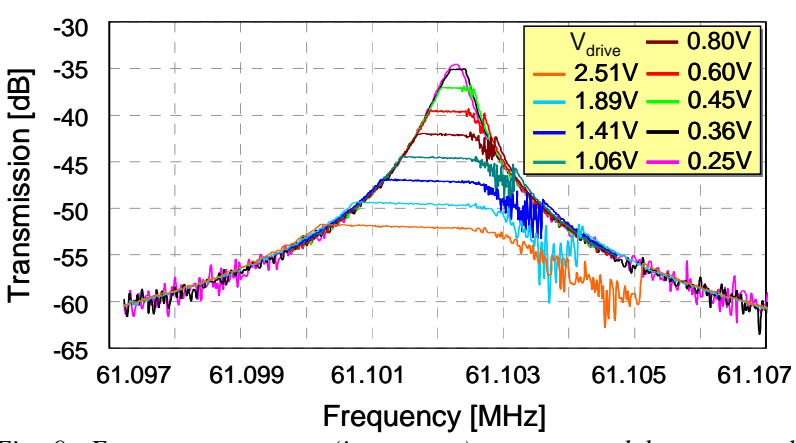

Fig. 9: Frequency response (in vacuum) as measured by a network analyzer of the capacitive version of the resoswitch for varying resonance input ac voltage amplitudes.

resistance $R_{p}$ and $3.5 \mathrm{pF}$ of capacitance, and that clearly matches the measured waveform.

Fig. 9 presents the swept frequency response for the capacitive resoswitch as measured via a network analyzer. Now, no dc electrical contact is made, so the transfer function flattens and reduces in gain value when switch impacting occurs, as expected. In addition, the bandwidth does indeed widen as the input voltage amplitude increases, clearly verifying nonlinear dynamical behavior.

To evaluate reliability, the resoswitch was operated continuously with $V_{P}=10 \mathrm{~V}$ for 75 hours ( 3 days or 16.5 trillion cycles) without failure at a frequency of $61 \mathrm{MHz}$, which is a frequency in the flat region of Fig. 8, and thus, a frequency where impacting occurs. Fig. 10 presents a plot of output amplitude versus time for the polysilicon version of the resoswitch. Although no failure is observed, degradation is seen, where after about 1.5 days, the output voltage begins to decrease significantly. Although 1.5 days corresponds to 7.7 trillion cycles at $61 \mathrm{MHz}$, which is more than

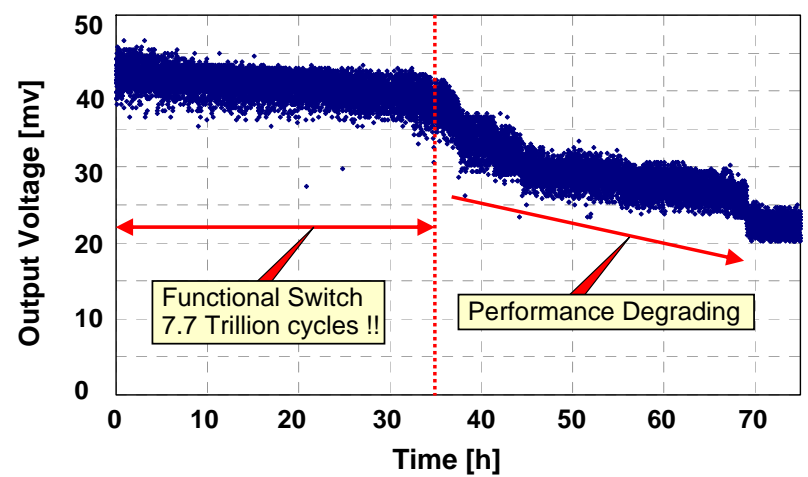

Fig. 10: Lifetime test of the polysilicon resoswitch

two orders of magnitude higher than the 100 billion cycles typically achieved by (good) RF MEMS switches, there is still cause for concern, here, since typical switched-mode power applications require much more than 3 days of operation. More study into the degradation mechanism is needed, but one possible reason for the observed degradation could be the growth of a thicker oxide or other dielectric on the switch contact interfaces. In the future, resoswitches constructed of metal with engineered contact surfaces will be investigated.

\section{CONCLUSIONS}

In this work, the resonance and nonlinear dynamical properties of a micromechanical wine-glass disk have been harnessed to demonstrate an impacting micromechanical switch with substantially higher switching speed, better reliability (even under hot switching), and lower actuation voltage, all by substantial factors, over existing RF MEMS switches. Although next generation versions of this resoswitch constructed in metal material [12] should be more widely applicable, the present polysilicon version can still find application to low current drain applications, such as Dickson charge pumps [3], where replacement of diodes with resoswitches should allow a very high output voltage, suitable for dc-biasing of capacitively transduced micromechanical resonators.

Acknowledgment: This work was supported by DARPA.

\section{REFERENCES}

[1] H. Kamitsuna, et al., "A fast low-power $4 \times 4$ switch IC using InP HEMTs ...,” Proceeding, IEEE CSIC, Jul. 2004, pp. 97-100.

[2] Y.-W. Lin et al., "60-MHz wine glass micromechanical ... oscillator," Tech. Dig., 2004 IEEE ISSCC, Feb. 15-19, 2004, pp. 322-323.

[3] J. F. Dickson, "On-chip high-voltage generation in NMOS ...," IEEE J. Solid-State Circuits, vol. 11, no.3, pp.374-378, June 1976.

[4] N.O. Sokal, et al., "Class E - A new class of high-efficiency tuned ...," IEEE J. Solid-State Circuits, vol. 10, no. 3, pp.168-176, June 1975.

[5] S. Wolf, Silicon processing for the VLSI era volume 3 - The submicron MOSFET. Sunset Beach, CA: Lattice Press, 1995.

[6] Agilent Technologies, "Agilent Solid State Switches-Selecting the right switch technology for your application,” 2007.

[7] D.A. Blackwell, et al., "X-band MMIC switch with ...," IEEE Microwave and Millimeter-Wave Monolithic Circuits Symposium, 1995.

[8] J. B. Muldavin, et al., "High-isolation inductively-tuned X-band ...," Tech. Dig., 2000 IEEE MTT-S, Boston, MA, pp. 169-172.

[9] S.; Lampen, et al., "A packaged, high-lifetime ohmic MEMS ...," Tech. Dig., 2003 IEEE MTT-S, pp. 1935-1938, vol.3, 8-13 June 2003

[10] G. M. Rebeiz, et al., "RF MEMS Switches and switch circuits," IEEE Microwave Magazine, Dec. 2001, pp. 59-71.

[11] J. Wang, et al., "1.51-GHz polydiamond ...,” Tech. Dig., 2004 IEEE MEMS Conf., Maastricht, The Netherlands, pp. 641-644.

[12] W.-L. Huang, et al., "Fully monolithic CMOS nickel micromechanical ...”, Tech. Dig., 2008 IEEE MEMS Conf., pp. 10-13. 